\title{
LA ACCESIBILIDAD A LA ACTIVIDAD COMERCIAL MINORISTA EN ÁREAS RURALES Y DESPOBLADAS: DIAGNÓSTICO Y DESAFÍOS PARA LA PLANIFICACIÓN
}

\author{
Aldo Arranz-López \\ Centro de Investigación del Transporte (TRANSyT). Universidad Politécnica de Madrid \\ aldo.arranz.lopez@upm.es \\ ORCID iD: http://orcid.org/0000-0002-6635-1386 \\ Julio A. Soria-Lara \\ Centro de Investigación del Transporte (TRANSYT). Universidad Politécnica de Madrid \\ julio.soria-lara@upm.es \\ ORCID iD: http://orcid.org/0000-0002-2383-6332 \\ Ricardo Badía-Lázaro \\ Departamento de Geografía y Ordenación del Territorio. Universidad de Zaragoza \\ rbadia@unizar.es \\ ORCID iD: http://orcid.org/0000-0003-0572-3681
}

Recibido: 29/07/2019; Aceptado: 22/11/2019.

Cómo citar este artículo/Citation: Arranz-López, Aldo; Soria-Lara, Julio A. y Badía-Lázaro, Ricardo (2020). La accesibilidad a la actividad comercial minorista en áreas rurales y despobladas: diagnóstico y desafíos para la planificación. Estudios Geográficos, 81 (288), e030. https://doi.org/10.3989/estgeogr.202045.025

RESUMEN: Tradicionalmente se ha prestado poca atención a conocer los efectos sociales de la accesibilidad a los principales destinos (ej. servicios y equipamientos básicos) en los espacios rurales, aislados y despoblados. Este artículo pretende abordar este desafío, estudiando la accesibilidad de distintos grupos de población al comercio minorista en áreas rurales despobladas. La comarca del Maestrazgo (Teruel, España), con una pérdida de población del 84\% entre 1900 y 2017, ha sido usada como caso de estudio. En primer lugar, se examinó la predisposición de la población para acceder al comercio diario y no diario a través de diferentes modos de transporte (coche, autobús, a pie). En segundo lugar, se utilizaron técnicas de agrupamiento para identificar distintos grupos socioeconómicos de población, y se analizaron estadísticamente sus funciones de caída de la demanda con el tiempo. Finalmente, se calculó y cartografió la accesibilidad multimodal al comercio minorista para los distintos grupos de población identificados previamente. Los resultados muestran niveles de accesibilidad bajos para el comercio diario y no diario, acentuando una alta dependencia del automóvil privado. Esto afecta particularmente a la población de edad más avanzada, con dificultades notables para tener disponibilidad de coche. El artículo concluye con algunas reflexiones sobre las implicaciones de los resultados obtenidos para la planificación territorial de áreas rurales y despobladas.

PALABRAS CLAVE: Transporte; despoblación; comercio; desigualdad; TIC; accesibilidad

\section{ACCESSIBILITY TO RETAIL IN RURAL AND DEPOPULATED PLACES: DIAGNOSIS AND CHALLENGES FOR PLANNING}

ABSTRACT: Academic literature has paid limited attention to the effects of accessibility variations in rural and depopulated areas. This paper aims to address this challenge by analyzing retail accessibility variations for different socio-economic groups of population, based on their transport mode preferences. The region of "The Maestrazgo", with a depopulation rate of $84 \%$ between 1900 and 2017, has served as case study. First, a questionnaire is administered focusing on the willingness of respondents to reach daily and non-daily retail by car, public transport, and on foot. Second, clustering techniques are used to identify different population groups, followed by the comparison of their distance-decay functions based on time-willingness to reach retail destinations. Third, relative accessibility maps based on gravity-based models are elaborated, highlighting places that exhibit statistical differences between the population groups. The results show low accessibility levels to daily and non-daily retail, which imply a high car dependency. Furthermore, accessibility for seniors is found to be significantly different than for the other groups. The paper closes with a discussion on how the obtained results can provide a platform to improve policies in depopulated areas.

KEY WORDS: Transport; depopulation; retail; inequalities; ICTs; relative accessibility

Copyright: @ 2020 CSIC. Este es un artículo de acceso abierto distribuido bajo los términos de la licencia de uso y distribución Creative Commons Reconocimiento 4.0 Internacional (CC BY 4.0). 


\section{INTRODUCCIÓN}

Incrementar los niveles de accesibilidad a los principales equipamientos y servicios se ha convertido en un elemento intrínseco de la vida cotidiana en las sociedades urbanas modernas (Bertolini, 2017). En cualquier área urbana occidental, es común encontrar que una misma persona realiza sus múltiples actividades diarias en múltiples localizaciones, sin que esto suponga una especial dificultad. Sin embargo, para los ciudadanos que residen en áreas rurales y despobladas, el acceso a ciertos equipamientos y servicios (ej. comercio de alimentación, servicios médicos) puede ser una barrera difícilmente salvable, originando problemas de cohesión e inclusión social a escala regional (McGrail y Humphreys, 2009; Nemet y Bailey, 2000; Orcao y Cornago, 2007; Shah, Milosavljevic, y Bath, 2017; van Leeuwen y Rietveld, 2011). Estas dificultades de accesibilidad a servicios y equipamientos pueden estar originadas por múltiples factores, por ejemplo: la existencia de una reducida oferta de servicios y actividades, dotación de transporte público limitado, el deficiente estado de las infraestructuras viarias/digitales y/o la dispersión de la población en el territorio (Arcury et al., 2005; Gray, Shaw, y Farrington, 2006; Jordan, Roderick, Martin, y Barnett, 2004; Yeager y Gatrell, 2014). Esta situación propicia que ciertos grupos de población (ej. personas de edad avanzada, familias con baja renta, personas con alguna discapacidad) dependan sistemáticamente de amigos y/o familiares para asegurar sus desplazamientos y acceder a los servicios y equipamientos que necesitan con cierta frecuencia.

A pesar de que existe un pensamiento creciente y optimista sobre la posibilidad de satisfacer parte de las necesidades de accesibilidad descritas a través de las Tecnologías de la Información y la Comunicación (TIC) (ej. comercio electrónico, teletrabajo, formación, ocio, sanidad, servicios sociales) (Zhen, Du, Cao, y Mokhtarian, 2018), una parte importante de la población de estos lugares rurales y despoblados sigue requiriendo de accesibilidad física a multitud de destinos. Pero incluso reconociendo el alto potencial de las TIC, tales espacios rurales y despoblados tienen problemas importantes en lo que respecta a la instalación de internet o en relación con la brecha digital ciudadana (Willis, 2019). En definitiva, es común encontrar que los residentes en áreas despobladas vean limitada su accesibilidad a equipamientos, trabajos y servicios, tanto física como virtualmente, lo que parcialmente contribuye a reforzar los procesos de despoblación existentes.
En base a las consideraciones descritas, este artículo se centra en analizar los niveles de accesibilidad multimodal (vehículo privado, transporte público, a pie) al comercio minorista en áreas rurales y despobladas. Para ello, busca responder a la siguiente pregunta: ¿Son similares los niveles de accesibilidad multimodal al comercio minorista para distintos grupos socioeconómicos en áreas rurales y despobladas? La respuesta a esta cuestión permitirá reflexionar sobre posibles acciones para la planificación territorial de estos lugares. En concreto, la comarca del Maestrazgo (Aragón, España) ha sido utilizada como laboratorio territorial por las siguientes tres razones: (i) Presenta una evolución intercensal negativa durante el pasado siglo, perdiendo el $85 \%$ de su población. (ii) Es un espacio lastrado por su escasez de actividad comercial, la cual ha disminuido drásticamente en las últimas décadas. (iii) Su localización geográfica le lleva a ser un espacio aislado, pero a su vez con cierta centralidad respecto de áreas altamente urbanizadas (ej. Valencia, Castellón, Teruel). En primer lugar, se diseñó y aplicó un cuestionario para examinar la predisposición de la población a acceder al comercio minorista mediante diferentes modos de transporte (coche, transporte público y a pie). En segundo lugar, se utilizó el algoritmo de k-modas para agrupar a la población en tres grupos homogéneos en función de sus características socioeconómicas. Finalmente, se estimó la accesibilidad multimodal para cada grupo aplicando un modelo gravitatorio. Los resultados de accesibilidad obtenidos para cada grupo de población fueron comparados mediante el uso de los estadísticos Kruskal-Wallis y Mann-Whitney.

El resto del documento se estructura de la siguiente manera. El apartado 2 presenta una revisión bibliográfica sobre el concepto de accesibilidad y sus aplicaciones en áreas rurales y despobladas. El apartado 3 describe el caso de estudio, mientras que el apartado 4 detalla el diseño metodológico realizado. El apartado 5 muestra los principales resultados obtenidos. El apartado 6 reflexiona sobre las implicaciones de estos resultados para la planificación territorial de las áreas rurales y despobladas. Finalmente, el apartado 7 concluye con algunas observaciones finales.

\section{LA ACCESIBILIDAD A DESTINOS: DESAFíos ACADÉMICOS EN ÁREAS RURALES DESPOBLADAS}

Se entiende por accesibilidad la capacidad de un individuo o grupo de individuos de acceder a multitud de destinos en un tiempo razonable, considerando 
los múltiples modos de transporte disponibles para ello (van Wee, 2016). Un aspecto predominante en los estudios sobre accesibilidad tanto en áreas urbanas como en áreas rurales y despobladas, es la visión de la accesibilidad a destinos como un valor absoluto que afecta por igual al conjunto de la población (Bocarejo y Oviedo, 2012; Yeager y Gatrell, 2014). Sin embargo, los niveles de accesibilidad de un individuo o conjunto de individuos están fuertemente ligados a aspectos como las preferencias individuales, los hábitos y costumbres, el contexto cultural, etc. En función de estos factores, la predisposición de cada individuo para cubrir el tiempo/distancia a cada destino usando un determinado modo de transporte es distinto, lo que refuerza la idea de la accesibilidad como un parámetro relativo para la planificación (Arranz-López, Soria-Lara, Witlox, y Páez, 2019; Morency, Paez, Roorda, Mercado, y Farber, 2011; Páez, Gertes Mercado, Farber, Morency, y Roorda, 2010). De este modo, concebir la accesibilidad desde un punto de vista relativo es clave para avanzar en la identificación de desigualdades sociales entre grupos de población, permitiendo el diseño de medidas que refuercen la accesibilidad a destinos de ciertos grupos socioeconómicos frente a otros. Inspirado por Páez et al. $(2010, p 3)$, la accesibilidad relativa puede definirse como el número de destinos (ej. comercios, servicios médicos, administración pública, trabajos) accesibles en un lugar específico para un individuo con unas características socioeconómicas determinadas, respecto de un individuo de un grupo de referencia que se encuentra en la misma localización.

De acuerdo a esta idea de accesibilidad relativa, en los últimos años se ha elevado considerablemente el número de investigaciones centradas en analizar los efectos de la accesibilidad a servicios básicos (i.e. alimentación, salud) para grupos específicos de población en áreas rurales y despobladas (principalmente la tercera edad). Sin embargo, se ha prestado poca atención a la comparación simultánea de los valores de accesibilidad entre varios grupos socioeconómicos. Además, otra limitación en el análisis de los niveles de accesibilidad en áreas despobladas es la falta de estudios que aborden una perspectiva multimodal de la accesibilidad, ya que mayoritariamente, los trabajos existentes se focalizan sobre la accesibilidad en coche (Shergold, Parkhurst, y Musselwhite, 2014; Yeager y Gatrell, 2014). Esto difiere de la idea de que la accesibilidad a servicios básicos puede quedar cubierta por diferentes modos de transporte, con efectos sociales distintos en función del grupo de población estudiado. Por ejemplo, en el contexto de las áreas despobladas donde la oferta de servicios es limitada, acceder a ellos supone generalmente una mayor dependencia del automóvil privado y del transporte público; mientras que en áreas urbanas con una mayor oferta de servicios, la accesibilidad a través de modos no motorizados puede ser mucho más decisiva (Reig Martínez, Goerlich Gisbert, y Catarino Martí, 2016). Por tanto, el rango de opciones para acceder a un lugar específico puede variar en función del tamaño del municipio, las infraestructuras de transporte, la dotación de transporte público, las características socioeconómicas de la población, sus hábitos y costumbres (Noguera Tur y Ferrandis Martínez, 2014). En el caso de esta investigación, se usa la noción de accesibilidad multimodal relativa como un aspecto clave para entender mejor los efectos sociales originados por los niveles existentes de accesibilidad a servicios en áreas rurales y despobladas (ej. identificar que grupos de población son más dependientes del automóvil privado).

\section{La Comarca del Maestrazgo}

El Maestrazgo se localiza en el sureste de Aragón (España). Lo componen un total de 15 municipios localizados en $1204 \mathrm{~km}^{2}$ (Figura 1). Su población ha decrecido intensamente durante el siglo XX perdiendo más de cuatro quintos del total. En concreto, la comarca ha pasado de 20600 habitantes a principios de siglo hasta los 3209 habitantes actuales (INE 2018a), lo que supone una densidad de población de 2,66 habitantes $/ \mathrm{km}^{2}$. Esta pérdida de población se remonta a la primera mitad del siglo XX, con un descenso de más de 7000 habitantes, que incluso llego a ser de 9000 en su punto álgido (1950-1970). Como resultado, la población en 1981 era de 4900 habitantes. Desde entonces, la tasa de despoblación ha disminuido constantemente. Actualmente, el porcentaje de población mayor de 65 años es especialmente elevado dentro del contexto español (29\%) (INE, 2018a).

La comarca del Maestrazgo no es sólo una de las regiones más despobladas de España, sino que fruto de este declive es también una de las más modestas en términos económicos (Romero y Escribano, 2015). El sector servicios está fuertemente vinculado al reciente aumento de la actividad turística en la región, que está dando lugar al $48 \%$ de los empleos existentes (Instituto Aragonés de Estadística, 2017). Por otro lado, el sector agrícola supone el $31 \%$ de tales empleos, una tasa particularmente alta en relación con el promedio español que es del 4,4\% (INE, 2018b). 
Este último sector, es la base de una industria agroalimentaria estructurada en torno a pequeñas empresas de carácter familiar y en las que predomina el autoempleo, dedicadas a la producción de queso, productos cárnicos, aceite y vino (Casulla, 2007). Estas actividades suponen prácticamente las únicas oportunidades laborales presentes en la zona.

Estos y otros factores de índole económicos (ej. cese de la actividad minera), unido a una escarpada topografía y a las deficientes comunicaciones (Romero y Pizarro, 2013) han influido fuertemente en su perdida poblacional. El Maestrazgo se localiza en el centro de un triángulo conformado por las autovías y autopistas que conectan Zaragoza, Valencia y Cataluña entre sí (Figura 1). Esta situación central refuerza su aislamiento al situarse lejos de dichas infraestructuras y, por lo tanto, en el límite exterior de las áreas de influencia de dichas ciudades. Las capitales provinciales más próximas son las ciudades de Teruel y Castellón. A nivel comarcal, la "falta de un núcleo rector, esto es, un núcleo central que organice el espacio" (Garrido y Faci, 2004) acentúa los desplazamientos a cabeceras comarcales próximas como Morella o Alcañiz. La red vial existente se encarga de dispersar el tráfico hacia regiones diferentes. El área sur, donde se encuentra Cantavieja, queda articulada por las carreteras A-226 y A-227 que facilitan el tráfico hacia Teruel y Castellón. El área noreste, cuyo muni-

FIgURA 1

LOCALIZACIÓN Y CARACTERÍSTICAS DEMOGRÁFICAS DE LA COMARCA DEL MAESTRAZGO

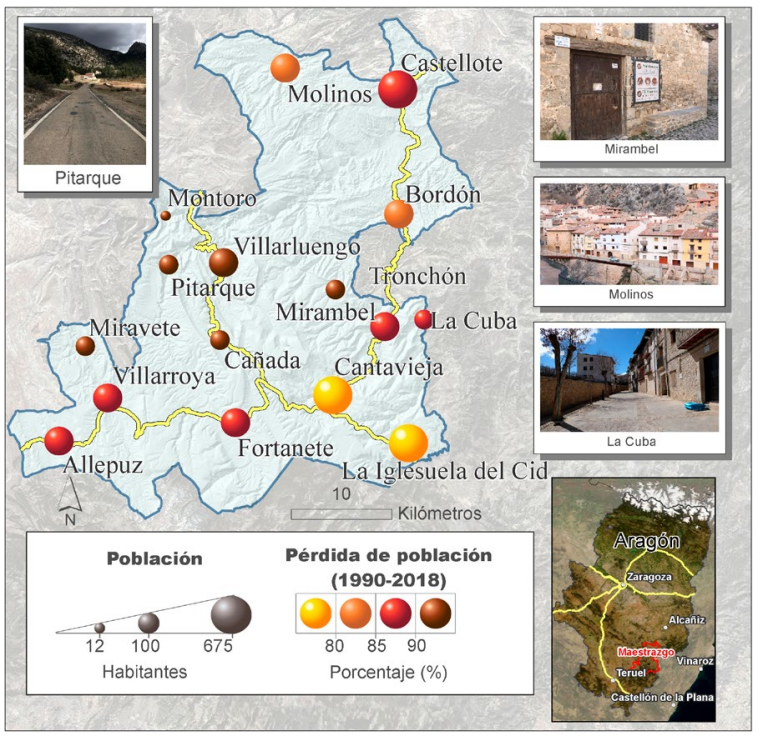

Fuente: IDE Aragón e INE (2018). Elaboración propia. cipio más poblado es Castellote, con 688 habitantes, queda articulada por la carretera A226 que comunica con el Bajo Aragón y la depresión del Ebro. Finalmente, el área noroeste, cuyo municipio más poblado (Villarluengo) tiene tan solo 169 habitantes, es atravesada por la carretera A1702 que comunica con la comarca de Andorra-Sierra de Arcos.

Dentro de la región, los servicios de transporte público son muy limitados e incluso inexistentes en gran parte de la comarca. En total existen tres rutas de autobús. La primera, de lunes a viernes, desde La Iglesuela del Cid hasta Teruel con una parada en Miravete de la Sierra (únicamente los jueves). La segunda, también de lunes a viernes, desde Cantavieja hasta Alcorisa, con una parada en Montoro de Mezquita (únicamente martes y viernes). La tercera, con origen en La Iglesuela del Cid y destino en Alcañiz, con servicio los martes y miércoles. Estas deficiencias en el servicio de transporte público han dado lugar a un aumento progresivo en el número de turismos desde el año 1999, pasando de 1200 a más de 1500 en 2016. Esto supone que el $88 \%$ de la población dispone de vehículo propio, bien un turismo o una furgoneta (Insitituto Aragonés de Estadística, 2018).

De manera similar al transporte público, la oferta de actividad comercial minorista en la comarca del Maestrazgo es bastante reducida, con un total de 34 tiendas clasificadas como comercio diario y 23 tiendas clasificadas como comercio no diario ${ }^{1}$. Ante la falta de comercio no diario, las cabeceras de las comarcas vecinas (Alcañiz y Morella) junto con Villafranca funcionan como centros básicos de este tipo de comercio. A mayor distancia, pero todavía con una incidencia importante sobre el Maestrazgo, se encuentran las localidades de Teruel, Castellón y Vinaroz que por su mayor tamaño demográfico poseen una mayor diversidad de comercio no diario. En 13 de los 15 municipios del ámbito de estudio se puede encontrar comercio diario, mientras que únicamente en 5 municipios se encuentra comercio no diario. Los municipios de mayor tamaño poblacional como Cantavieja, Castellote y La Iglesuela del Cid ofertan comercio de ambos tipos. El ejemplo más representativo es Cantavieja con 7 tiendas clasificadas como comercio diario y otras 7 como comercio no diario. Finalmente, cabe destacar que los municipios más pequeños como Allepuz y Miravete de la Sierra no cuentan con ningún tipo establecimiento comercial, aunque si con puestos de comercio itinerante (ej. alimentación, ropa) algunos días a la semana. A pesar de la importancia estos mercados itinerantes para los municipios más pequeños, éstos no han sido 
incluidos en los análisis dado que no cuentan con un espacio físico fijo al que la población pueda acudir cualquier día de la semana.

\section{Metodología}

El diseño metodológico para dar respuesta a la pregunta de investigación planteada en la introducción del artículo consistió en tres fases (Figura 2): (i) ad- quisición de datos; (ii) técnicas de agrupamiento y análisis de funciones de caída de la demanda con el tiempo; (iii) cálculo y cartografiado de la accesibilidad relativa multimodal al comercio minorista.

\section{Adquisición de datos}

La fuente principal de información fue una encuesta en la que se preguntó acerca de la predisposición de

FIGURA 2

ESQUEMA METODOLÓGICO

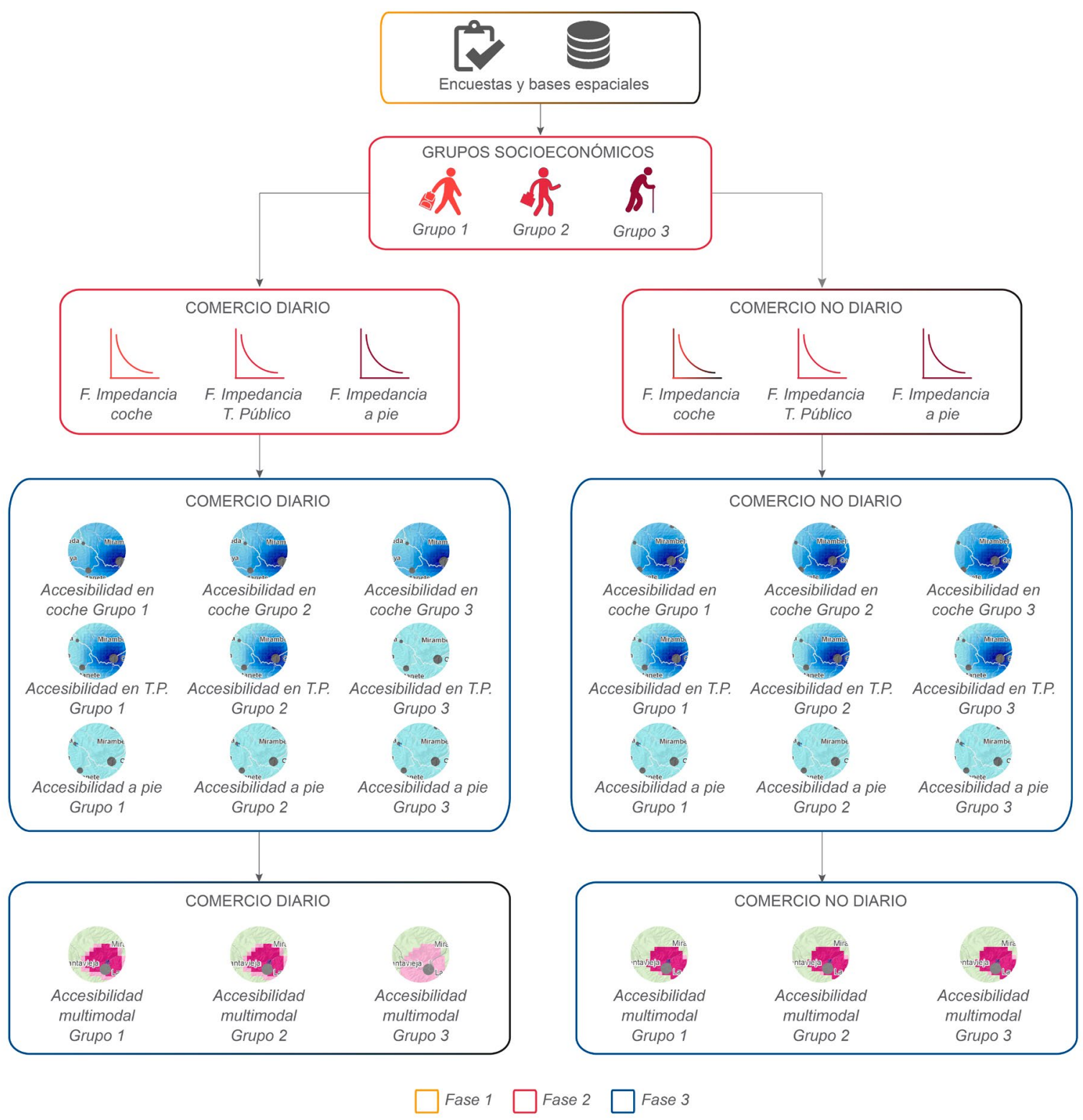

Fuente: Elaboración propia. 
la población del Maestrazgo a acceder al comercio diario y no diario, a través de tres modos de transporte: (i) coche; (ii) transporte público; (iii) a pie. En total, 96 personas accedieron a contestar la encuesta, mayoritariamente de manera presencial y ocasionalmente online (en el caso de los municipios más grandes con buen servicio de internet). Las encuestas presenciales fueron cruciales en el proceso de obtención de datos, dado el alto porcentaje de población mayor que vive en el Maestrazgo, con una brecha digital importante. La selección de los participantes se hizo mediante la organización de tres campañas de campo en marzo de 2018.

La encuesta, que contenía un total de 17 preguntas fue dividida en dos bloques. El primer bloque incluyó ocho preguntas relacionadas con las características socioeconómicas y demográficas de los encuestados, tales como la edad, el género, municipio de residencia, nivel de formación, situación laboral, renta neta del hogar, disponibilidad de carné de conducir y disponibilidad de coche en el hogar familiar. Las respuestas de género, situación laboral y disponibilidad de carné de conducir y/o coche se codificaron en respuestas binarias. Las respuestas para el nivel de formación y renta neta del hogar se codificaron en intervalos. El nivel de formación incluyó las siguientes categorías: sin educación reglada, baja (educación primaria); media (educación secundaria) y alta (estudios de grado y superiores). Para la renta mensual del hogar se establecieron tres intervalos: baja $(<1000 €)$, media (1000$2000 €)$ y alta (>2000€). El segundo bloque de la encuesta incluyó un total de 9 preguntas relacionadas con la predisposición de la población encuestada a acceder a la actividad comercial minorista diaria y no diaria en coche, transporte público y caminando. Las respuestas se codificaron en intervalos de $15,30,45$, $60,90,120,150$ y 180 minutos para coche y transporte público; y 5, 10, 15, 30, 45, 60 minutos para acceder a pie. La pregunta tipo que se realizó fue: ¿cuánto tiempo está predispuesto a invertir para acceder a los establecimientos comerciales no diarios en coche / transporte público / a pie?

En cuanto al proceso de selección y tamaño de la muestra, existen una serie de limitaciones que pueden originar sesgos en los resultados obtenidos. En primer lugar, la muestra no ha sido aleatorizada debido al pequeño tamaño de los municipios, que haría necesario encuestar a un porcentaje muy elevado de población para conseguir una muestra representativa. La dificultad de obtener una muestra más grande y completamente aleatorizada es aún mayor si se tiene en cuenta que en algunos de los municipios de la comarca del Maestrazgo, no toda la población empadronada reside de manera habitual en dicha región, haciendo difícil que una persona seleccionada aleatoriamente esté presente en el momento de realizar el cuestionario. A modo de ejemplo, el municipio de Cañada de Benatanduz cuenta con 42 habitantes censados de los cuales, según la propia corporación municipal, sólo 18 viven de forma permanente en el pueblo. En segundo lugar, y dado que las encuestas se realizaron a pie de calle, la población más mayor que no participa de la vida social del municipio no quedó adecuadamente representada en la muestra.

Además de los datos procedentes de la encuesta, también se utilizaron cinco bases de datos espaciales, incluyendo una malla vectorial regular de $1 \mathrm{~km}$ de lado, la actividad comercial, la red de carreteras y la red de calles de cada municipio (Tabla 1). Para la geo-

TABLA 1

BASES DE DATOS ESPACIALES

\begin{tabular}{|c|c|c|c|c|c|}
\hline \multicolumn{2}{|c|}{ Base de datos } & Fuente & Clase de entidad & Año & Finalidad \\
\hline 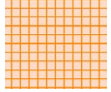 & $\begin{array}{l}\text { Malla vectorial de } 1 \times 1 \\
\mathrm{~km}\end{array}$ & $\begin{array}{l}\text { Agencia Europea de } \\
\text { Medioambiente }\end{array}$ & Polígono & & $\begin{array}{c}\text { Representar los cálculos } \\
\text { de accesibilidad }\end{array}$ \\
\hline & Red de carreteras & $\begin{array}{c}\text { Centro Nacional de } \\
\text { Información Geográfica }\end{array}$ & Línea & 2018 & $\begin{array}{c}\text { Calcular la matriz } \\
\text { origen-destino }\end{array}$ \\
\hline & $\begin{array}{l}\text { Actividad commercial } \\
\text { diaria y no diaria }\end{array}$ & $\begin{array}{l}\text { Digitalizadas manualmente } \\
\text { durante las campañas de } \\
\text { campo }\end{array}$ & Punto & & $\begin{array}{c}\text { Calcular la accesibilidad } \\
\text { para cada tipología } \\
\text { comercial }\end{array}$ \\
\hline
\end{tabular}

Fuente: Elaboración propia. 
localización de los establecimientos comerciales, el equipo de investigación visitó todos los municipios de la comarca y realizó un inventario de todos los establecimientos comerciales, clasificándolos en las dos categorías mencionadas en la descripción del caso de estudio: diario y no diario.

\section{Técnicas de agrupamiento y análisis de funciones de caída de la demanda con el tiempo}

En esta fase metodológica se identificaron grupos homogéneos de población en base a sus características socioeconómicas. Para ello, se utilizó el algoritmo de K-modas, específicamente desarrollado para trabajar con variables de naturaleza categórica (Chaturvedi, Green, y Caroll, 2001). La implementación de estas técnicas de agrupamiento se debe, en gran medida, a las características y limitaciones de la muestra previamente indicados (ver apartado 4.1), lo que limita el proceso de inferencia lógica mediante el uso de modelos econométricos. En total, siete variables de la encuesta fueron analizadas para los análisis de agrupamiento: género, edad, nivel de formación, situación laboral, renta mensual del hogar y disponibilidad de carné de conducir y/o coche.

En primer lugar, se realizó un análisis de correspondencia múltiple (ACM) para explorar las relaciones entre las siete variables socioeconómicas incluidas en el cuestionario. Este proceso mostró que la edad, la renta mensual del hogar, la situación laboral y la disponibilidad de coche en el hogar familiar eran las variables más débilmente relacionadas y, por lo tanto, las que fueron utilizadas para el análisis de agrupamiento. El algoritmo de k-modas, incluido en el paquete KlaR del software $\mathrm{R}$, permitió obtener tres grupos socioeconómicos de población. El número óptimo de grupos se determinó en base a el método de silueta y utilizando la función fviz_nbclust en RStudio.

Finalmente, se estimaron y analizaron las funciones de caída de la demanda con el tiempo para cada grupo de población previamente identificado. Tales funciones de caída de la demanda con el tiempo representan la predisposición (en minutos) de un individuo o grupo de individuos para desplazarse físicamente hasta el comercio minorista usando un modo de transporte determinado. Estas curvas de caída de la demanda con el tiempo suelen tener una forma exponencial negativa, que indica que cuánto más cerca (en minutos) está el comercio minorista del individuo, más predisposición hay a desplazarse físicamente hasta este destino (Figura 3). La comparación de las curvas de caída de la demanda con el tiempo de los distintos grupos de población identificados previamente se basó en las pruebas estadísticas de KruskalWallis y Mann-Whitney. La prueba no paramétrica de Kruskal-Wallis se utilizó para comparar de manera simultánea las funciones de caída de la demanda con el tiempo de todos los grupos poblacionales identificados. Diferencias significativas con un nivel $p<0,05$ indicarían que al menos la predisposición a acceder en coche, transporte público o a pie de uno de los grupos de población era estadísticamente diferente a la de los otros grupos. La prueba no paramétrica de Mann-Whitney se utilizó para analizar las funciones por pares de grupos, en el caso de que se hubieran obtenido diferencias estadísticamente significativas para la prueba de Kruskal-Wallis. Estas funciones de caída de la demanda con el tiempo serían la base para realizar los cálculos de accesibilidad al comercio diario y no diario para cada grupo de población.

FIGURA 3

\section{EJEMPLO DE FUNCIÓN DE CAÍDA DE LA DEMANDA} CON EL TIEMPO

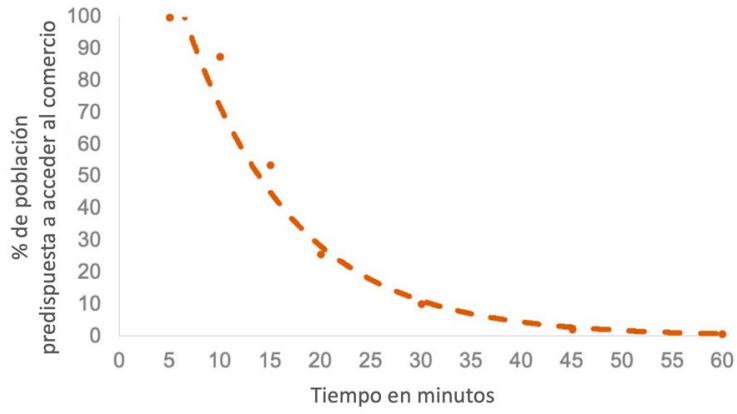

Fuente: Elaboración propia.

\section{Cálculo y cartografiado de la accesibilidad relativa multimodal}

Para calcular y cartografiar la accesibilidad relativa multimodal de los diferentes grupos socioeconómicos de población se utilizó un modelo gravitatorio. Los modelos gravitatorios consideran la accesibilidad de una zona determinada (un píxel de $1 \times 1 \mathrm{~km}$ en este estudio) como la suma del coste de viaje de todas las zonas del área de estudio, ponderada por el número de establecimientos comerciales en el destino y una función de caída de la demanda con el tiempo que 
representa la predisposición, en minutos, de la población para acceder a dichos establecimientos comerciales por medio de un modo específico de transporte. La fórmula utilizada fue la siguiente:

$$
A_{i}=\sum E_{i \neq j} e^{\beta X_{i j}}
$$

donde: $A_{i}$ es la accesibilidad para la zona $i ; E_{j}$ es el número de establecimientos comerciales en cada destino $j ; X_{i j}$ es la distancia entre el origen y el destino, y ? es un parámetro constante que varía para cada grupo de población y que refleja la predisposición a acceder al comercio diario y no diario de la población a través de los distintos modos de transporte analizados. Según se indicó anteriormente, las funciones de caída de la demanda con el tiempo para los tres modos adoptaron una función exponencial negativa (Figura 3), comúnmente utilizada en las investigaciones de transporte (Handy y Niemeier, 1997; lacono, Krizek, y El-Geneidy, 2010; Vega, 2012).

La distancia entre los orígenes y potenciales destinos fue calculada en minutos a través de una matriz origen-destino (MOD). Por un lado, la estimación de la velocidad para el coche y transporte público se hizo en base al límite máximo de velocidad de cada vía de comunicación. Por otro lado, la velocidad caminando se estimó en $4 \mathrm{~km} / \mathrm{h}$ de media (Marquet y Miralles-Guasch, 2014). En el caso de la MOD para el coche, cada celda del área de estudio fue incluida como origen y como destino, mientras que para el transporte público la MOD sólo incluyó aquellas cel- das donde se ubicaba una parada de autobús. Finalmente, para la MOD a pie se calculó el tiempo de viaje desde cada celda al municipio más cercano, aplicando un análisis de ubicación-asignación.

Con el objetivo de representar los resultados de accesibilidad para cada grupo socioeconómico, se utilizó un cubo comparativo de accesibilidad multimodal (Silva, 2013) (Figura 4), mediante el cual se establecieron ocho categorías discretas que van desde "baja accesibilidad al comercio" por cualquier modo de transporte, hasta "alta accesibilidad al comercio" mediante los tres modos de transporte incluidos en el estudio (coche, transporte público y a pie).

\section{RESULTADOS}

\section{Características de la muestra}

Aproximadamente, el mismo número de hombres y de mujeres respondieron el cuestionario, siendo la edad media de la muestra 50,44 años. En cuanto al nivel de formación, el $14,4 \%$ declaró no tener estudios, el 25,8\% educación básica, el 31,9\% estudios medios y el $27,8 \%$ estudios superiores. Un $64 \%$ de la población encuestada estaba trabajando, mientras que el $36 \%$ estaba desempleado o jubilado. Las rentas más comunes fueron las de $1,000 €$ o menos $(44,3 \%)$, así como las de entre $1,000 €$ y $2,000 €(46,4 \%)$. Sólo el $9,3 \%$ de la muestra declaró tener una renta del

FIGURA 4

\section{CUBO DE ACCESIBILIDAD MULTIMODAL}

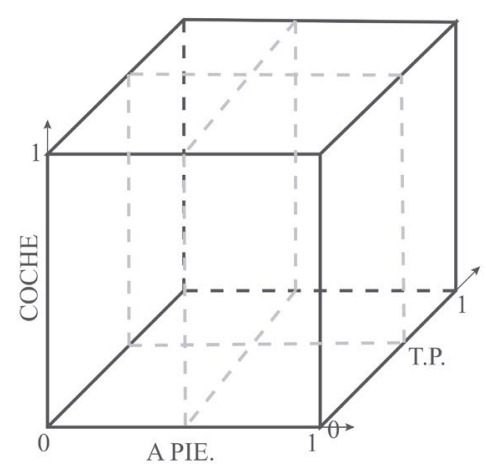

Comercio poco accesible en todos los modos de transporte

Comercio accesible caminando y poco accesible en coche y en T. Público Comercio accesible en coche y poco accesible caminando y en T. Público

Comercio accesible en coche y caminando y poco accesible en T. Público
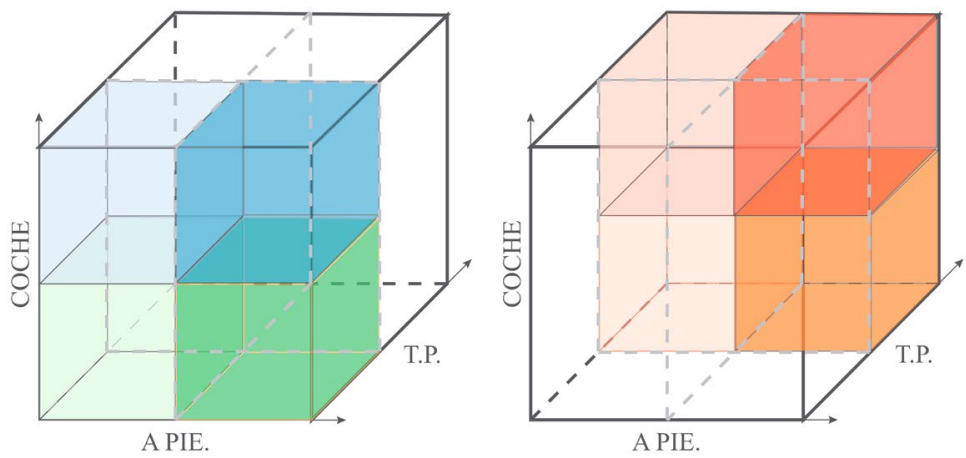

Comercio accesible T. Público poco accesible en coche y caminando Comercio accesible caminando y en T. Público y poco accesible en coche Comercio accesible en coche y T. Público y poco accesible caminando Comercio accesible en todos los modos de transporte

Fuente: Elaboración propia. 
hogar superior a los 2,000€. Finalmente, hay que destacar que la mayoría de la población encuestada tenía carné de conducir $(83,5 \%)$ y disponibilidad de coche en su hogar $(84,5 \%)$.

\section{Grupos socioeconómicos y funciones de caída de la demanda con el tiempo}

De acuerdo a las técnicas de agrupamiento realizadas (apartado 4.2), se identificaron un total de tres grupos socioeconómicos de población en el caso de estudio (Figura 5), para los que la predisposición a acceder al comercio diario y no diario es diferente, $y$ que queda representada por la constante $b$ (Tabla 2) descrita en la fórmula de accesibilidad (apartado 4.3).

- Jóvenes empleados de alta renta (grupo \#1-35 individuos): en este grupo se incluyen individuos entre 18 y 39 años, con trabajo, disponibilidad de coche y una renta mensual entre $1,000 €$ y $2,000 €$.
- Adultos empleados de baja renta (grupo \#2-31 individuos): compuesto por individuos entre 40 y 64 años, con disponibilidad de coche y una renta mensual inferior a los $1,000 €$.

- Tercera edad (grupo \#3-30 individuos): formado por individuos de 65 años y más, jubilados, sin disponibilidad de coche y con una renta mensual inferior a los 1,000€.

Una vez identificados tales grupos socioeconómicos, se compararon estadísticamente sus funciones de caída de la demanda con el tiempo. La prueba estadística de Kruskal-Wallis, mostró diferencias estadísticamente significativas $(p<0,05)$ entre las curvas de caída de la demanda con el tiempo de los tres grupos socioeconómicos para el acceso en coche y transporte público a la actividad comercial minorista diaria (Tabla 3). Esto significa que al menos uno de los grupos socioeconómicos tenía una predisposición (en minutos) significativamente diferente al resto de grupos a acceder en coche o transporte público al comercio diario.

FIGURA 5

CARACTERÍSTICAS DE LOS GRUPOS DE POBLACIÓN IDENTIFICADOS
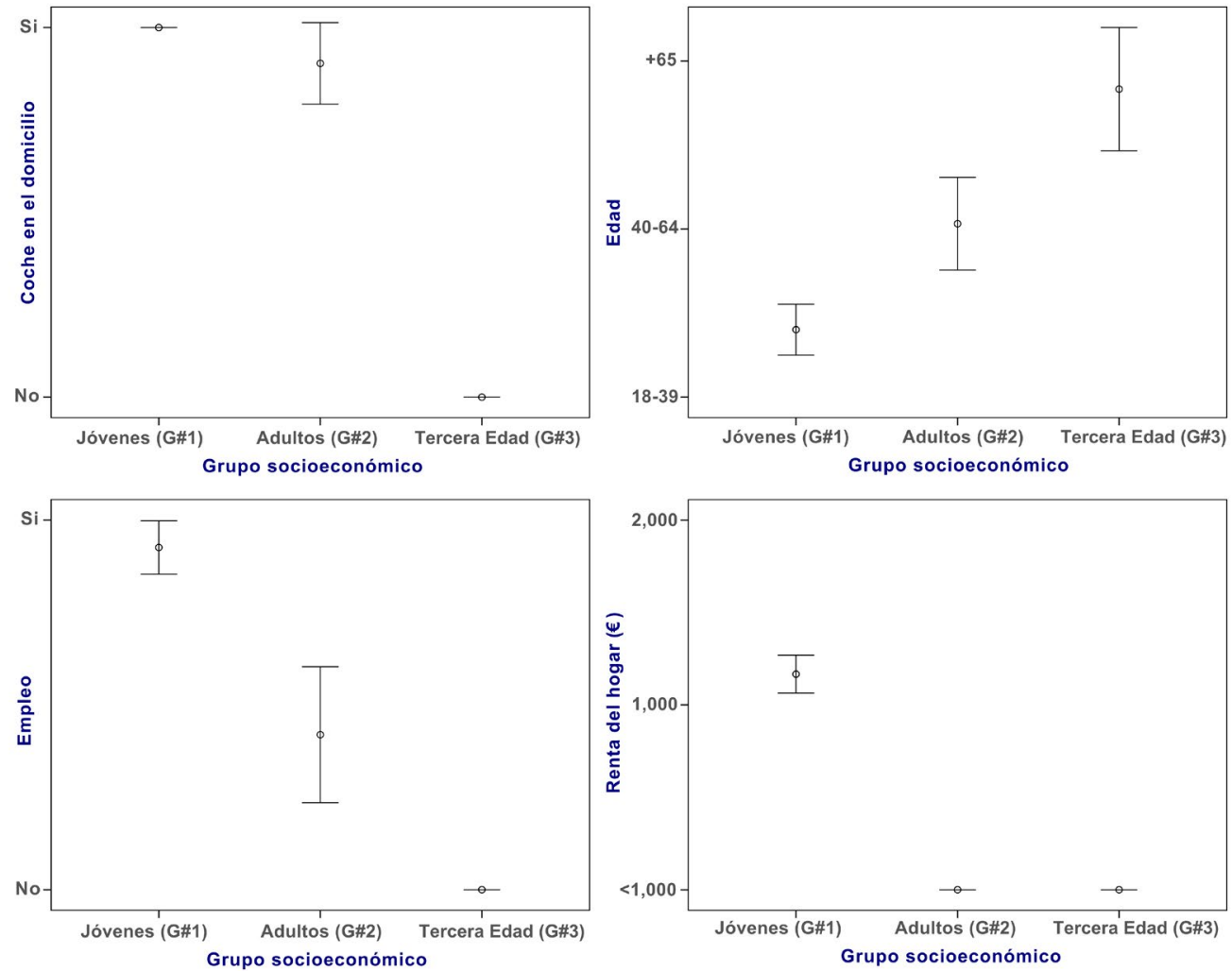

Fuente: Elaboración propia. 
TABLA 2

PARÁMETROS $\beta$ PARA CADA GRUPO DE POBLACIÓN, MODO DE TRANSPORTE Y TIPOLOGÍA COMERCIAL

\begin{tabular}{|l|l|c|c|c|}
\hline & & Grupo \#1 & Grupo \#2 & Grupo \#3 \\
\hline \multirow{4}{*}{$\begin{array}{l}\text { Comercio } \\
\text { diario }\end{array}$} & Coche & $-0,077$ & $-0,075$ & $-0,046$ \\
\cline { 2 - 5 } & $\begin{array}{l}\text { Transporte } \\
\text { público }\end{array}$ & $-0,073$ & $-0,074$ & $-0,060$ \\
\cline { 2 - 5 } & Caminando & $-0,222$ & $-0,222$ & $-0,24$ \\
\hline \multirow{3}{*}{$\begin{array}{l}\text { Comercio } \\
\text { no diario }\end{array}$} & $\begin{array}{l}\text { Transporte } \\
\text { público }\end{array}$ & $-0,050$ & $-0,048$ & $-0,058$ \\
\cline { 2 - 5 } & Caminando & $-0,075$ & $-0,063$ & $-0,063$ \\
\cline { 2 - 5 } & & & $-0,223$ \\
\hline
\end{tabular}

Fuente: Elaboración propia.

TABLA 3

TEST DE KRUSKAL-WALLIS PARA LAS FUNCIONES DE CAÍDA DE LA DEMANDA DEL COMERCIO DIARIO Y NO DIARIO POR MÚLTIPLES MODOS

\begin{tabular}{|l|c|c|c|}
\hline Función de impedancia & Coche & Transporte público & A pie \\
\hline Comercio diario & $\mathbf{0 , 0 0 0}$ & $\mathbf{0 , 0 0 0}$ & 1 \\
\hline Comercio no diario & 0,913 & $\mathbf{0 , 0 0 0}$ & 1 \\
\hline
\end{tabular}

Fuente: Elaboración propia.

Tanto el tamaño de los municipios como la ubicación de la actividad comercial pueden ayudar a comprender mejor los resultados obtenidos para la prueba de kruskal-Wallis. Por un lado, que los municipios sean tan pequeños en extensión, hace que todos los grupos puedan acceder a pie sin problemas a los establecimientos comerciales diarios (en el caso de que los hubiera), reduciéndose así las opciones de obtener diferencias estadísticamente significativas. Por otro lado, el bajo número de comercios no diarios en la comarca (en su mayoría ubicados en los municipios de Cantavieja y Castellote), combinado con un servicio de transporte público deficiente, obliga a la mayoría de los residentes a usar un automóvil para llegar a tiendas minoristas no diarias, reduciendo la posibilidad de encontrar diferencias significativas para las curvas de caída de la demanda con el tiempo relativas a la predisposición a viajar en coche a dicho tipo de comercios.

Para aquellos valores estadísticamente significativos en la prueba de Kruskal-Wallis, se aplicó la prueba estadística de Mann-Whitney. Esto permitiría identificar diferencias estadísticamente significativas entre pares de grupos socioeconómicos (Tabla 4). Los re- sultados muestran que, la predisposición (en minutos) de las personas mayores para acceder al comercio diario en coche y transporte público, es significativamente diferente de los otros grupos. Estas diferencias podrían ser debidas a que el grupo de la tercera edad, en general, no utiliza el coche para sus desplazamientos, mientras que los jóvenes empleados de alta renta y los adultos empleados de baja renta lo utilizan habitualmente. Además, las personas mayores declararon durante las campañas de campo una predisposición nula para acceder al comercio en transporte público, a diferencia de los otros grupos que si mostraron cierta predisposición a utilizar el transporte público.

TABLA 4

TEST DE MANN-WHITNEY POR COCHE Y TRANSPORTE PÚBLICO

\begin{tabular}{|c|c|c|c|c|c|c|}
\hline \multicolumn{7}{|c|}{ Cunción de impedancia coche } \\
\hline & G\#1 & G\#2 & G\#3 \\
\hline G\#1 & - & 0,012 & \multicolumn{2}{c|}{$\mathbf{0 , 0 0 0}$} \\
\hline G\#2 & - & - & \multicolumn{2}{c|}{$\mathbf{0 , 0 0 0}$} \\
\hline \multicolumn{7}{|c|}{ Función de impedancia Transporte público } \\
\hline & \multicolumn{2}{|c|}{ Comercio diario } & \multicolumn{2}{c|}{ Comercio no diario } \\
\hline & G\#1 & G\#2 & G\#3 & G\#1 & G\#2 & G\#3 \\
\hline G\#1 & - & $\mathbf{0 , 0 0 0}$ & $\mathbf{0 , 0 0 0}$ & - & 0,306 & $\mathbf{0 , 0 0 0}$ \\
\hline G\#2 & - & - & $\mathbf{0 , 0 0 0}$ & - & - & $\mathbf{0 , 0 0 0}$ \\
\hline
\end{tabular}

Fuente: Elaboración propia.

\section{Cálculo y cartografía de la accesibilidad multimodal relativa}

Teniendo en cuenta que la investigación analiza la accesibilidad a dos tipologías comerciales (diario y no diario) mediante tres modos de transporte (coche, transporte público y a pie), para cada grupo de población se obtuvieron un total de seis funciones de caída de la demanda con el tiempo que serían la base para calcular la accesibilidad. Para ello se utilizó el modelo gravitatorio detallado en el apartado 4 . El resto del apartado detalla los resultados de accesibilidad para cada modo de transporte y tipología comercial.

La accesibilidad en coche a la a actividad comercial diaria mostró valores más altos para los grupos de jóvenes empleados con alta renta y adultos emplea- 
dos con baja renta (Figura 6). Los municipios de Cantavieja, Castellote y La Iglesuela del Cid mostraron los niveles más altos de accesibilidad, ya que es donde se localizan la mayoría de los establecimientos comerciales del Maestrazgo. El resto de la comarca obtuvo unos valores medios o bajos de accesibilidad, lo que podría ser indicativo de que una parte importante de su población se desplaza a otros municipios fuera de la comarca para cubrir sus necesidades comerciales. Por el contrario, los niveles de accesibilidad en coche para el grupo de la tercera edad fueron los más bajos de todo el caso de estudio. Para este grupo, los valores más altos se localizaron principalmente en Cantavieja, mientras que el resto de los municipios presentaron valores medios o bajos. Estas variaciones de accesibilidad entre los grupos se deben principalmente a la distinta predisposición de acceder al comercio minorista mediante coche. Este hecho queda reflejado en las opiniones que algunos de los encuestados hicieron durante las campañas de campo. Cabe destacar como los jóvenes empleados de alta renta y adultos empleados de baja renta declararon de manera unánime que, aunque utilizan las tiendas de comercio diario existentes en su localidad, generalmente se desplazan cada dos o tres semanas a los municipios de Cantavieja y Castellote, e incluso a otros subcentros funcionales fuera de la comarca, donde existe una mayor especialización y diversidad comercial, como Alcañiz, Teruel o Castellón. De esta forma, tanto los jóvenes empleados con rentas altas como los adultos empleados con rentas bajas afirmaron que "su vida diaria depende del coche para cubrir la mayoría de sus necesidades comerciales".

En el caso del grupo de población de tercera edad, declararon comprar casi exclusivamente en los comercios de su propio municipio. En aquellos municipios en los que no hay tiendas físicas, suelen existir mercados itinerantes semanales que los miembros de este grupo de población utilizan para satisfacer sus necesidades. Analizando los datos resultantes de la encuesta esto es consecuencia, en muchos casos, de no tener carné de conducir. Además, a pesar de no incluir preguntas específicas relacionadas con la salud o limitaciones físicas en la encuesta, muchas personas incluidas en este grupo declararon tener problemas de salud y limitaciones físicas asociadas a la edad. Por ello, algunos de los encuestados afirmaron que normalmente realizan encargos a sus familiares o vecinos cuando éstos se desplazan hasta municipios de mayor tamaño y con mayor dotación comercial. En definitiva, el grupo de la tercera edad se encuentra claramente en una situación de desven- taja en comparación con los grupos de jóvenes empleados de alta renta y adultos empleados de baja renta. En este sentido, el potencial cierre de la única tienda que hay abierta, y no todos los días, en los municipios más pequeños como Pitarque, Bordón o La Cuba, aumenta el riesgo de exclusión social para la población más envejecida.

La accesibilidad en coche al comercio no diario (Figura 7) mostró valores similares para los tres grupos analizados. Si bien los municipios de Cantavieja, Castellote y La Iglesuela de Cid presentaron los valores más altos, el área oeste del caso de estudio mostró los valores más bajos de accesibilidad debido a la inexistencia de establecimientos comerciales no diarios en esos espacios. Las pequeñas variaciones de accesibilidad entre grupos se deben principalmente a dos factores. Por un lado, la limitada oferta de comercio no diario en toda la comarca. Por otro lado, la limitada oferta de transporte público.

Los valores de accesibilidad más altos en transporte público al comercio diario fueron para los grupos de jóvenes empleados con alta renta y adultos empleados con baja renta (Figura 6). Sin embargo, el grupo de la tercera edad presentó una accesibilidad nula ya que las respuestas del cuestionario reflejaron que no estaban dispuestos a acceder al comercio diario en transporte público. En este caso, Cantavieja presentó los valores más altos de accesibilidad, mientras que el resto de la comarca mostró una accesibilidad nula en transporte público. Esto reafirma la escasa oferta y mal servicio de transporte público en toda la comarca. Se debe destacar aquí que un valor "cero" de accesibilidad para la población más mayor en transporte público, la hace completamente dependientes de la escasa actividad comercial diaria de su municipio de residencia.

En el caso de la accesibilidad en transporte público al comercio no diario, los tres grupos socioeconómicos mostraron patrones espaciales similares (Figura 7) con valores especialmente bajos en toda la comarca. De nuevo Cantavieja, Castellote y la Iglesuela del Cid presentaron los valores más altos de accesibilidad. No obstante, la baja predisposición a acceder en transporte público es debido al mal funcionamiento de éste y los elevados tiempos de desplazamiento. Este último factor, quedó evidenciado durante las campañas de campo, en las que los grupos de jóvenes empleados con alta renta y adultos empleados con baja renta declararon que "rara vez cogemos el autobús para llegar al comercio no diario, ya que supone casi el doble de tiempo que en coche". Por otra parte, 
FIGURA 6

ACCESIBILIDAD RELATIVA AL COMERCIO DIARIO
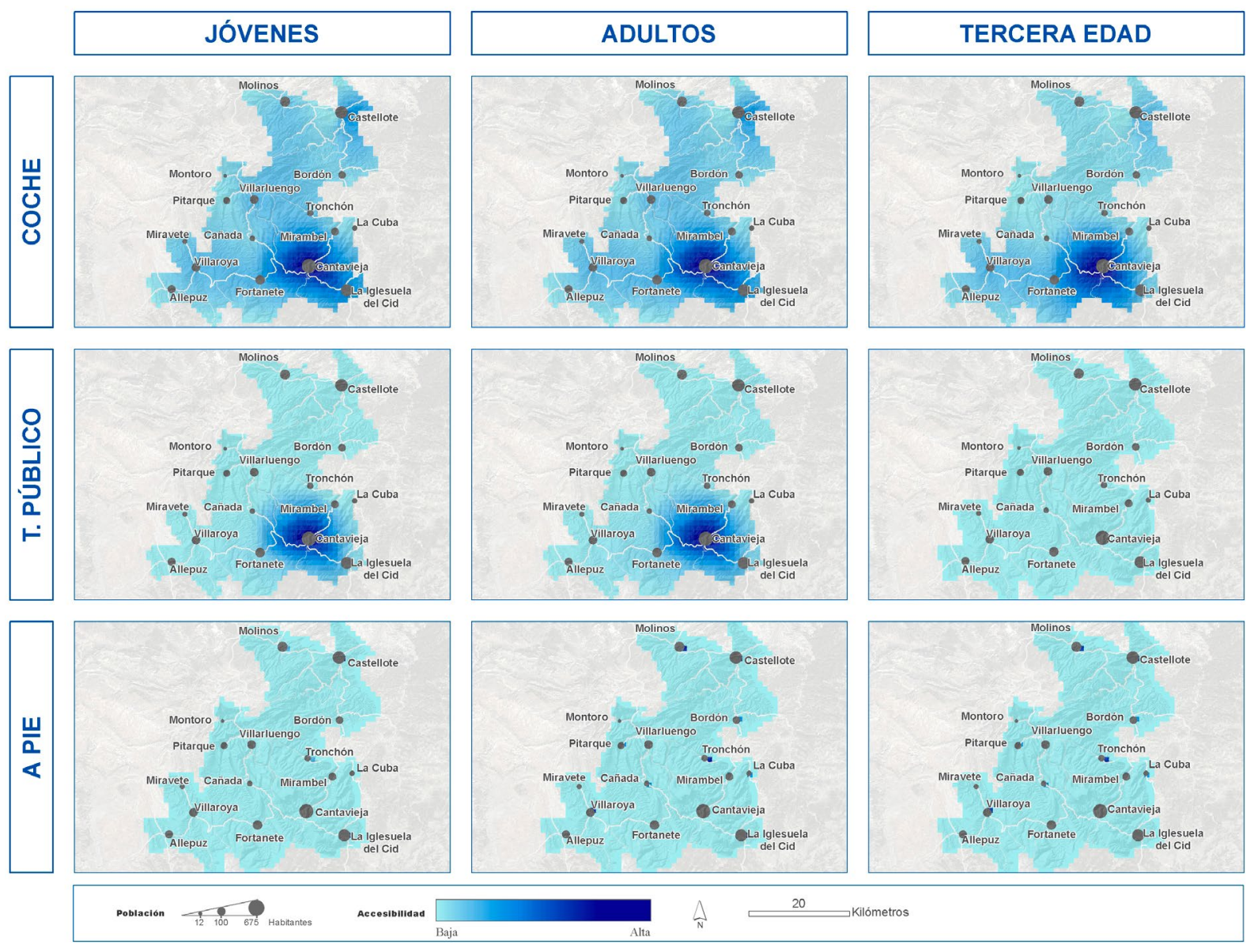

Fuente: IDE Aragón y trabajo de campo. Elaboración propia.

el grupo de la tercera edad presentó los valores más altos, lo que significa una mayor predisposición para acceder al comercio no diario. No obstante, esto contrasta con la realidad que vive este grupo de población, ya que los participantes en el estudio reconocieron de manera unánime que "los horarios que tiene no son los más convenientes para las personas más mayores, ya que tendríamos que pasar todo el día fuera de casa. Nos gustaría poder ir por la mañana pronto y poder volver a comer a casa".

En el caso de la accesibilidad a pie al comercio diario (Figura 6), sólo los núcleos de población tuvieron valores superiores a "cero". Cantavieja, Castellote y La Iglesuela del Cid fueron los municipios con los valores más altos para los tres grupos socioeconómicos, mientras que los adultos y los mayores de 65 años presentaron los valores más altos para el resto de los municipios (i.e. Molinos, Bordón,
Villarroya de los Pinares). Los bajos valores de accesibilidad a pie en los municipios más pequeños se deben principalmente a la escasa, e incluso nula, oferta de comercio diario. Por ejemplo, algunos municipios sólo contaban con una tienda y con horario limitado (ej. martes y viernes) que vendía productos de muy primera necesidad (principalmente comestibles). Incluso en los municipios más pequeños, el bar hacía las veces de tienda. Esto hace que los productos que se venden tengan un precio más elevado que en los establecimientos ubicados en municipios más poblados. Entre otras razones, esto se debe al aislamiento geográfico de la comarca y las malas condiciones de la infraestructura viaria, que elevan el costo del servicio de transporte. La suma de todos estos factores serían la razón por los que la población de los municipios más pequeños prefiere desplazarse en coche hasta los municipios más 
FIGURA 7

ACCESIBILIDAD RELATIVA AL COMERCIO NO DIARIO

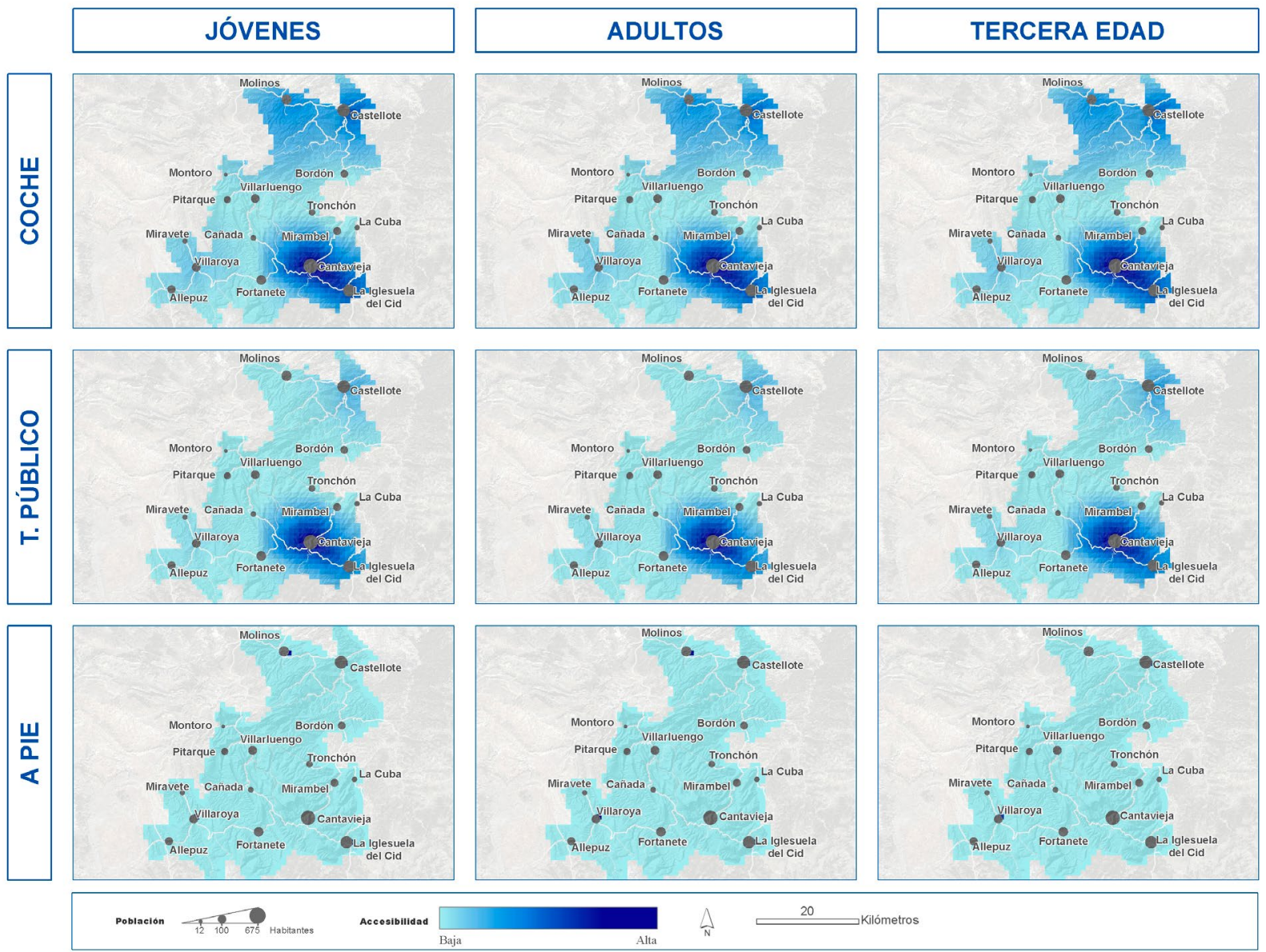

Fuente: IDE Aragón y trabajo de campo. Elaboración propia.

grandes, donde existe una oferta comercial superior y con unos precios más bajos. Por consiguiente, la cohorte de personas mayores de 65 años se ve nuevamente desfavorecida, dados sus menores ingresos y su acceso limitado al coche.

En el caso de la accesibilidad a pie al comercio no diario (Figura 7), la situación es aún más preocupante. Sólo los municipios de Cantavieja, Castellote y La Iglesuela del Cid presentan una oferta real de comercio no diario accesible a pie para un número importante de habitantes. Si bien es cierto que municipios como Molinos, donde se localizan dos peluquerías, presentan valores altos de accesibilidad, se puede afirmar que la oferta comercial minorista no diaria no existe realmente en este municipio.

En la última parte del análisis, los valores de accesibilidad por los tres modos y para cada grupo so- cioeconómico se combinaron mediante el uso del cubo de evaluación de accesibilidad. Aunque se establecieron un total de ocho categorías potenciales de accesibilidad multimodal (ver Sección 3 y Fig. 4), solo seis de ellas estaban presentes en el caso de estudio.

Por un lado, las tipologías de accesibilidad multimodal al comercio diario fueron muy similares para los tres grupos analizados (Figura 8). Casi toda el área de estudio tenía "baja accesibilidad a la actividad comercial minorista", mientras que el municipio de Cantavieja mostró una "alta accesibilidad al comercio mediante modos de transporte motorizados". La diferencia más notable se encontró para el grupo de la tercera edad, que presentó valores más altos para las celdas con "alta accesibilidad al comercio en coche". 
FIGURA 8

ACCESIBILIDAD MULTIMODAL AL COMERCIO DIARIO Y NO DIARIO

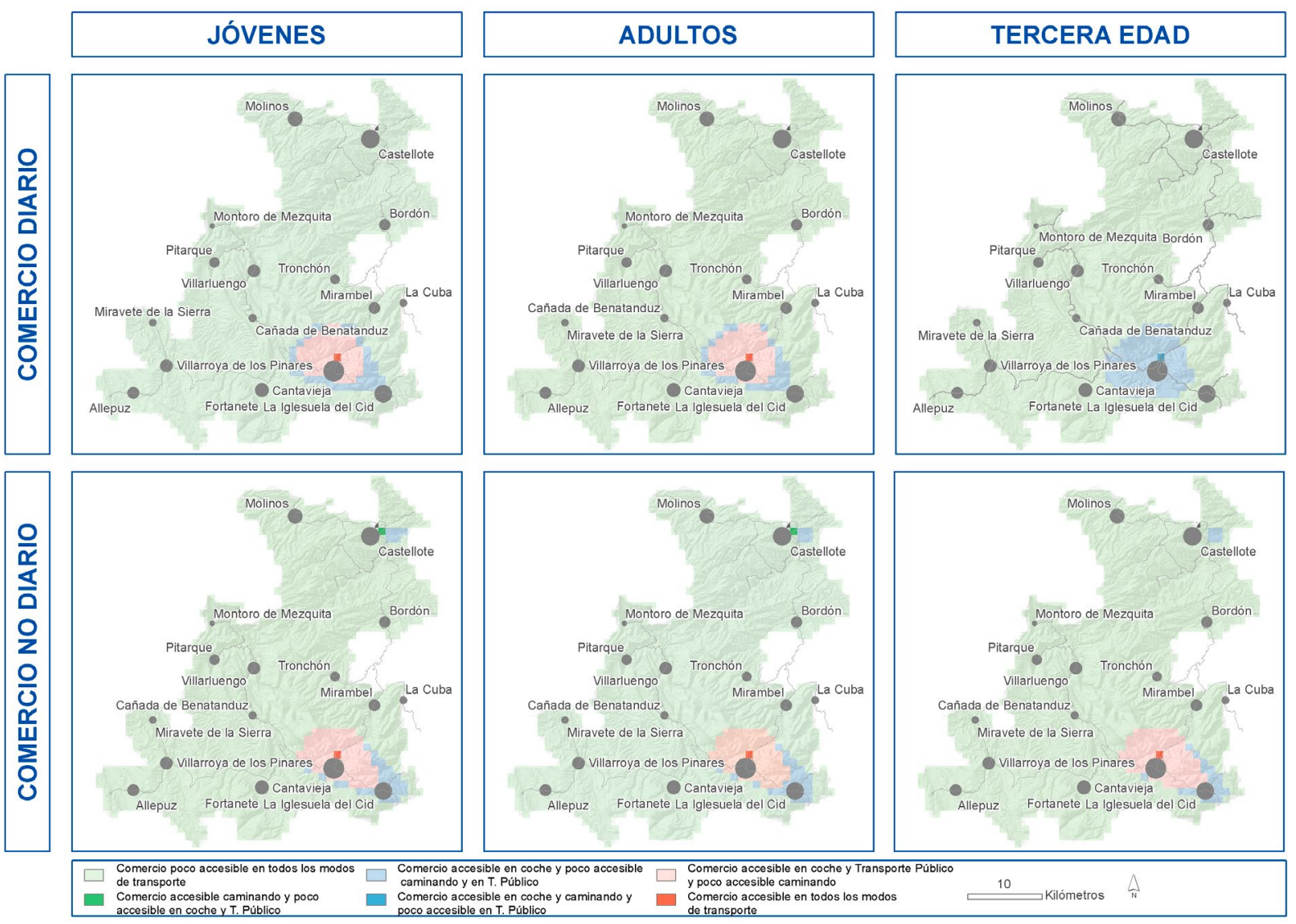

Fuente: IDE Aragón y trabajo de campo. Elaboración propia.

Por otro lado, las tipologías de accesibilidad multimodal al comercio no diario fueron muy similares para todos los grupos de población (Figura 8). En el municipio de Cantavieja, los tres grupos socioeconómicos encontraron "alta accesibilidad al comercio mediante modos de transporte motorizados". En el caso de La Iglesuela del Cid, solo se encontró "comercio altamente accesible en coche". Finalmente, el municipio de Castellote tuvo "alta accesibilidad al comercio en coche " $\mathrm{y}$ "baja accesibilidad al comercio en transporte público" para los tres grupos de población.

\section{IMPLICACIONES PARA LA PLANIFICACIÓN TERRITORIAL}

Abordar cómo maximizar los niveles de accesibilidad a los principales destinos en áreas rurales y despobladas es uno de los principales desafíos a nivel de planificación territorial, para un adecuado desa- rrollo regional de estos lugares. El éxodo de población rural hacia áreas urbanas está generando un fuerte crecimiento de las grandes metrópolis, dando lugar a situaciones de desigualdad social acentuada y bajos estándares de sostenibilidad ambiental. Además, los procesos de despoblación también pueden intensificar gravemente las consecuencias del cambio climático, en la medida y tienden a sufrir un mayor riesgo de incendio, una reducción en los flujos de los ríos y una mayor erosión del suelo (Lasanta, Nadal-Romero, y Arnáez, 2015). En consecuencia, la academia debe proporcionar soluciones para mejorar las conexiones locales y regionales, así como la investigación debería de poner un mayor acento en la desigualdad y sostenibilidad a escala regional (Soria-Lara y Banister, 2017).

En base a lo anterior y tomando como fundamento los resultados obtenidos en esta investigación, se analizarán detalladamente tres alternativas potenciales para aumentar los niveles de accesibilidad a la acti- 
vidad comercial minorista: (i) servicios de transporte bajo demanda, (ii) el papel de las TIC y (iii) la planificación conjunta de la ciudad y su área de influencia.

- Servicios de transporte bajo demanda: la oferta limitada de transporte público es un problema importante en la comarca del Maestrazgo. En particular, la población más mayor, más dispuesta a usar el transporte público, está drásticamente afectada por el bajo número de concesiones, servicios e itinerarios existentes. En este contexto, donde la demanda de transporte público es baja, la implementación de soluciones de servicios de transporte bajo demanda podría ser particularmente efectiva. En este sentido, la comarca del Maestrazgo junto con Gúdar-Javalambre (Aragón, España) están potenciando el transporte en el medio rural a través del trámite de licencias de taxi y subvenciones para la compra de vehículos por parte de particulares. Las potenciales soluciones incluyen el diseño de itinerarios de transporte público compartido, donde los niños pueden ir hasta la escuela y simultáneamente la población residente al comercio minorista; servicios de transporte personalizados puerta a puerta bajo la noción de la movilidad como servicio (MaaS); o, finalmente, servicios de coche compartidos para cubrir los viajes diarios a la actividad comercial, equipamientos sanitarios y otras necesidades básicas. No obstante, parte de la movilidad del grupo de tercera edad ya se está dando de este modo en el Maestrazgo, pero generando viajes ad hoc que dependen de familiares y amigos. Sin embargo, los incentivos de políticas para soluciones de transporte bajo demanda podrían generar modelos de negocio entre los más jóvenes, que deberían ser impulsados por la administración pública con el fin de reducir el riesgo de exclusión social de algunos grupos de población. Tales soluciones, deberían incluir entregas a domicilio de productos de alimentación y otros bienes de primera necesidad dentro de la comarca y otros servicios de movilidad.

- El papel de las TIC: a través de múltiples vías, las TIC están cada día más integradas en nuestra sociedad. Por ejemplo, muchas personas pueden trabajar, comprar o acceder a formación a través de internet. Uno de los mayores problemas es que las TIC son ignoradas en el proceso de planificación de la accesibilidad. Además, los estu- dios que analizan la capacidad de las TIC como un instrumento transformador en áreas despobladas son todavía limitados (Carrasco, Miller, y Wellman, 2008; Páez y Scott, 2007; van Wee, 2016). Tres problemas básicos impiden que las TIC mejoren el acceso a la actividad comercial en el contexto de áreas rurales o despobladas como el Maestrazgo. Primero, la calidad del servicio de internet se ve periódicamente afectado por averías o un servicio deficiente, a pesar de las repetidas promesas por parte de la administración de mejorar la calidad del servicio en áreas rurales y despobladas. Por ejemplo, la fibra óptica y la conexión $4 \mathrm{G}$ son prácticamente inexistentes dentro del caso de estudio de esta investigación. En segundo lugar, un importante porcentaje de la población de la comarca son personas en la tercera edad, que nunca han utilizado internet o un smartphone. Por lo tanto, una parte importante de mejorar la accesibilidad basándose en las TIC debe centrarse en reducir la brecha digital para la población del Maestrazgo y, específicamente, para la población más envejecida. En tercer lugar, organizar el comercio minorista en torno a las TIC requiere resolver problemas clave de logística. En el contexto del Maestrazgo, con una topografía de montaña, este es un desafío crucial dado el estado deficiente de muchas de sus infraestructuras de transporte. El desarrollo y promoción del nexo TIC-Comercio podría derivar en un nuevo modelo de negocio en el que los establecimientos sirvieran productos ad-hoc al consumidor en función de sus necesidades en cada momento. No obstante, para ello sería necesario una previa asociación de los comerciantes (ej. cooperativa) para hacerlo viable económica y logísticamente. La inversión en infraestructuras sería la medida principal, pero también se deberían explorar otras vías como la promoción de centros logísticos o proyectos piloto de entregas a través de vehículos aéreos no tripulados (drones).

- Planificar conjuntamente la ciudad y su área de influencia: parte de los problemas de accesibilidad en las áreas despobladas están relacionados con la visión inherente de esos lugares como autosuficientes. Un argumento recurrente es que cada región debería tener una amplia oferta comercial minorista, servicios de atención médica, etc. Sin embargo, conseguir ser autosuficiente en una región como el Maestrazgo, con una dinámica demográfica regresiva en la que el 33\% 
de sus municipios no llega a los 50 habitantes, resulta especialmente difícil. Por ello, en estos pequeños núcleos debería fomentarse el proyecto "Multiservicio rural" que la cámara de Comercio de Teruel lleva potenciando desde 2003, y que ha permitido la apertura y mantenimiento de establecimientos multiservicio que permiten aglutinar en un mismo local actividades de comercio minorista junto con otras de restauración e incluso turísticas. Entre sus ventajas, están el ahorro de costes derivado de realizar varias actividades en un espacio, una mayor afluencia de clientes al atraer a personas que de otro modo no hubieran acudido al comercio, así como, la posibilidad de trabajar en red con otro tipo de profesionales del entorno inmediato, lo que facilita diversificar intermediarios o distribuidores (Escribano et al, 2015). El resto de necesidades, de carácter ocasional, podrían ser cubiertas por las áreas urbanas más próximas, principalmente Alcañiz para los municipios localizados al norte del Maestrazgo (ej. Molinos, Castellote) y Teruel y Castellón (ej. Vinaroz) para los municipios del sur (ej. Cantavieja, Mirambel). Es decir, la planificación conjunta de la ciudad y su área de influencia podría fomentar soluciones de accesibilidad basadas en la proximidad para determinadas necesidades (i.e. actividad comercial diaria en pequeñas tiendas o establecimientos multiservicio), reforzando las conexiones entre las áreas despobladas y urbanas cuando sea conveniente (i.e. actividad comercial no diaria). No obstante, una dificultad comentada en el apartado 3 es la abrupta orografía que condiciona el trazado de las infraestructuras y por tanto la calidad de las conexiones entre los municipios del Maestrazgo y los centros funcionales más próximos. Además, una de las principales barreras en el contexto español para implementar un proceso de planificación que integre la ciudad y su área de influencia se encuentran en la falta de una definición y precisa de las áreas de influencia rural de los entornos urbanos (Feria-Toribio, 2018; Pascual Rosa, Aguilera-Benavente, y Salado García, 2019). Otro problema importante es el actual sistema legal de planificación territorial y urbana, en el que es difícil introducir instrumentos de planificación que combinen simultáneamente áreas urbanas y sus regiones.

\section{CONCLUSIONES}

Este artículo ha tratado de aportar conocimiento adicional al debate sobre la accesibilidad a los servicios básicos en áreas despobladas. En particular, se ha centrado en estudiar como la accesibilidad multimodal a la actividad comercial minorista varía entre tres grupos socioeconómicos de población: jóvenes empleados de alta renta, adultos empleados de baja renta y tercera edad. La accesibilidad multimodal para la población en la tercera edad es significativamente diferente a la de los otros dos grupos socioeconómicos, siendo muy dependientes del coche para acceder al comercio no diario, además de presentar limitaciones para acceder a pie al comercio diario. Por el contrario, los jóvenes y adultos presentan valores más altos de accesibilidad al comercio diario y no diario, dada la mayor disponibilidad de coche, así como una mayor predisposición para desplazarse hasta otros municipios incluso fuera de la comarca del Maestrazgo. La comarca cuenta con dos municipios donde se concentran los valores más altos de accesibilidad: Cantavieja y Castellote. Ambos municipios presentan las tasas más bajas de despoblación y actúan como nodos principales de los desplazamientos en transporte público.

El artículo concluye con algunas reflexiones de las implicaciones para la planificación territorial, detallando tres estrategias que podrían incrementar los niveles de accesibilidad a la actividad comercial minorista: (i) servicios de transporte bajo demanda; (ii) el papel de las TIC; (iii) planificar conjuntamente la ciudad y su área de influencia. Aunque estas posibles soluciones se presentan para el caso específico del Maestrazgo, todas podrían ser extrapoladas a otros contextos espaciales. La necesidad de proyectos piloto para probar tales soluciones es crucial en el contexto español y europeo, dadas la crítica situación de despoblación que sufren un número creciente de territorios. Un elemento importante para el diseño de tales soluciones debe ser la manera en que las características socioeconómicas de la población pueden afectar de manera drástica a la efectividad de las intervenciones a realizar. Esto queda reflejado en los resultados de este artículo, donde se ha demostrado como los niveles de accesibilidad son relativos a cada grupo de población, así como dependientes de las normas culturales y hábitos en el contexto de las áreas rurales despobladas. En el caso del Maestrazgo, el gobierno regional y los encargados de formular políticas en el corto, medio y largo plazo deben asumir escenarios futuros donde la tasa de población disminuirá y la proporción de personas mayores será aun mayor. 
Finalmente, la investigación futura sobre accesibilidad a la actividad comercial minorista debería basarse en la comparación de los resultados obtenidos en otras áreas despobladas en diferentes contextos geográficos (i.e. municipios despoblados más cercanos a grandes áreas urbanas), con el fin de profundizar en como la geografía afecta a los niveles de accesibilidad, así como a las posibles soluciones a los desafíos que ésta plantea. Para ello, sería necesario adaptar y superar algunas cuestiones metodológicas planteadas en esta investigación. En primer lugar, se podrían incluir los mercados itinerantes en los cálculos de accesibilidad, en función de su importancia en el área de estudio. No obstante, esto llevaría a realizar un estudio de la accesibilidad en el espacio-tiempo, ya que el comercio itinerante no está disponible cualquier día de la semana. En segundo lugar, se debería adaptar el grid ( $1 \times 1 \mathrm{~km}$ en este estudio) con el objetivo de analizar la accesibilidad dentro de cada municipio si su tamaño y actividad comercial existente así lo requiriese. En tercer lugar, sería necesario obtener una muestra representativa de la población, con el fin de evitar sesgos en los resultados obtenidos, así como en el proceso de inferencia lógica de la investigación presentada. Sin embargo, se debe tener en cuenta que, dado el pequeño tamaño de los municipios, conseguir una muestra representativa

\section{NOTAS}

1 El comercio diario incluye panaderías, fruterías, carnicerías, pescaderías, supermercados y ultramarinos. El comercio no diario incluye papelerías,

\section{REFERENCIAS BIBLIOGRÁFICAS}

Arcury, T. A., Gesler, W. M., Preisser, J. S., Sherman, J., Spencer, J., y Perin, J. (2005). The effects of geography and spatial behavior on health care utilization among the residents of a rual region. Health Services Research, 40 (1), 135-155. https://doi. org/10.1111/j.1475-6773.2005.00346.x

Arranz-López, A., Soria-Lara, J. A., Witlox, F., y Páez, A. (2019). Measuring relative non-motorized accessibility to retail activities. International Journal of Sustainable Transportation, 13(9), 639-651. https://doi.org/10.1080/15568318.2018.1498563

Bocarejo S., J. P., y Oviedo H., D. R. (2012). Transport accessibility and social inequities: a tool for identification of mobility needs and evaluation of tiene un coste temporal y económico elevado. Otras líneas de investigación podrían basarse en explorar el potencial de las TIC para acceder a la actividad comercial minorista, así como sus efectos para diferentes grupos de población, también comparando los municipios mejor localizados frente a aquellos localizados más remotamente.

\section{Agradecimientos}

Este artículo se ha realizado en el marco de los siguientes proyectos de investigación:

- "TRANS-URBAN: Simulación de escenarios colaborativos para integrar políticas de transporte urbano sostenible y usos del suelo", financiado por el Plan Nacional de Investigación I+D+i (20182020). Referencia: CSO2017-86914-C2-2-P.

- iCITIES: Efectos urbanos y sociales del uso de internet para compras y trabajo". Acción financiada por la Comunidad de Madrid en el marco del Convenio Plurianual con la Universidad Politécnica de Madrid en la línea de actuación estímulo a la investigación de jóvenes doctores. Referencia: M190020074JASL

farmacias, peluquerías, hostelería y talleres mecánicos. No se han tenido en cuenta los mercados itinerantes.

transport investments. Journal of Transport Geography, 24, 142-154. https://doi.org/10.1016/j. jtrangeo.2011.12.004

Carrasco, J. A., Miller, E. J., y Wellman, B. (2008). How Far and with Whom Do People Socialize? Transportation Research Record: Journal of the Transportation Research Board, 2076(1), 114-122. https://doi.org/10.3141/2076-13

Casulla, B. (2007) El renacer del sector agroalimentario en el Maestrazgo. En J. Ibañez. (Coord.), Comarca del Maestrazgo (pp. 237-246). Zaragoza, España: Publicaciones electrónicas del Gobierno de Aragón. 
Chaturvedi, A., Green, P. E., y Caroll, J. D. (2001). K-modes Clustering. Journal of Classification, 18(1), 35-55. https://doi.org/10.1007/s00357-001-0004-3

Escribano Pizarro, J., Esparcia Pérez, J., y Serrano Lara, J.J. (2015) El comercio en los espacios rurales valencianos: caracterización, funciones, problemáticas y estrategias de actuación. Cuadernos Geográrficos, 54(1), 87-112.

Estadística, Insitituto Aragonés de. (2018). Estadística Local de Aragón. Ficha territorial. Comarca: Maestrazgo.

Estadística, Instituto Aragones de. (2017). Estadística Local. Trabajo, Salarios y Relaciones Laborales. Afiliaciones en alta a la Seguridad Social.

Feria-Toribio, J. M. (2018). Los procesos metropolitanos en España. Intensificación estructural y nuevos desafíos. Papers: Regió Metropolitana de Barcelona: Territori, Estratègies, Planejament, 1(61), 28-40.

Garrido, J., y Faci, Y. (2004) Causas de la despoblación en la cuenca del río Guadalope. Comarcas del Bajo Aragón y Maestrazgo. Propuestas de políticas demográficas y de desarrollo endógeno. Informe CEDDAR, 1.

Gray, D., Shaw, J., y Farrington, J. (2006). Community transport, social capital and social exclusion in rural areas. Area, 38(1), 89-98. https://doi. org/10.1111/j.1475-4762.2006.00662.x

Handy, S. L., y Niemeier, D. A. (1997). Measuring Accessibility: An Exploration of Issues and Alternatives. Environment and Planning A: Economy and Space, 29(7), 1175-1194. https://doi.org/10.1068/ a291175

lacono, M., Krizek, K. J., y El-Geneidy, A. (2010). Measuring non-motorized accessibility: issues, alternatives, and execution. Journal of Transport Geography, 18(1), 133-140. https://doi.org/10.1016/j. jtrangeo.2009.02.002

INE. (2018a). Cifras oficiales de población de los municipios españoles: Revisión del Padrón Municipal.

INE. (2018b). Encuesta de población activa, Trimestre 1-2018. Resultados Nacionales. Distribución porcentual de los ocupados por sector económico y sexo.

Jordan, H., Roderick, P., Martin, D., y Barnett, S. (2004). Distance, rurality and the need for care: access to health services in South West England. International Journal of Health Geographics, 3(21), 1-9. https://doi.org/10.1186/1476-072X-3-21

Lasanta, T., Nadal-Romero, E., y Arnáez, J. (2015). Managing abandoned farmland to control the impact of re-vegetation on the environment. The state of the art in Europe. Environmental Science and Policy, 52, 99-109. https://doi.org/10.1016/j. envsci.2015.05.012

Marquet, O., y Miralles-Guasch, C. (2014). Walking short distances. The socioeconomic drivers for the use of proximity in everyday mobility in Barcelona. Transportation Research Part A: Policy and Practice, 70, 210-222. https://doi. org/10.1016/j.tra.2014.10.007

McGrail, M. R., y Humphreys, J. S. (2009). Measuring spatial accessibility to primary care in rural areas: Improving the effectiveness of the two-step floating catchment area method. Applied Geography, 29(4), 533-541. https://doi.org/10.1016/j.apgeog. 2008.12.003

Morency, C., Paez, A., Roorda, M. J., Mercado, R., y Farber, S. (2011). Distance traveled in three Canadian cities: Spatial analysis from the perspective of vulnerable population segments. Journal of Transport Geography, 19(1), 39-50. https:// doi.org/10.1016/j.jtrangeo.2009.09.013

Nemet, G. F., y Bailey, A. J. (2000). Distance and health care utilization among the rural elderly. Social Science y Medicine, 50(9), 1197-1208. https:// doi.org/10.1016/S0277-9536(99)00365-2

Noguera Tur, J., y Ferrandis Martínez, A. (2014). Accesibilidad y provisión de servicios de interés general en las áreas rurales de la Unión Europea: un análisis a partir del eurobarómetro. Boletín de La Asociación de Geógrafos Españoles, 64, 377404. https://doi.org/10.21138/bage.1703

Orcao, A. I. E., y Cornago, C. D. (2007). Accessibility to basic services in one of the most sparsely populated areas in Europe: the province of Teruel (Spain). Area, 39(3), 295-309. https://doi.org/10.1111/ j.1475-4762.2007.00749.x

Páez, A., Gertes Mercado, R., Farber, S., Morency, C., y Roorda, M. (2010). Relative Accessibility Deprivation Indicators for Urban Settings: Definitions and Application to Food Deserts in Montreal. Urban Studies, 47(7), 1415-1438. https://doi. org/10.1177/0042098009353626 
Páez, A., y Scott, D. M. (2007). Social Influence on Travel Behavior: A Simulation Example of the Decision to Telecommute. Environment and Planning A: Economy and Space, 39(3), 647-665. https://doi.org/10.1068/a37424

Pascual Rosa, V., Aguilera-Benavente, F., y Salado García, M. J. (2019). Clasificación y análisis de los procesos de cambio en la forma urbana de las áreas metropolitanas españolas: aplicación de métricas espaciales. Estudios Geográficos, 80(286), 006. https://doi.org/10.3989/estgeogr.201926.006

Reig Martínez, E., Goerlich Gisbert, F. J., y Catarino Martí, I. (2016). Delimitación de áreas rurales y urbanas a nivel local. Demografía, coberturas del suelo y accesibilidad.

Romero Renau, L., Pizarro, J. E. (2013) Diagnóstico y propuestas para la revitalización de las sierras de Teruel (Gúdar-Javalambre y Maestrazgo). Ager, Revista de Estudios sobre Despoblación y Desarrollo Rural, 15, 115-152. https://doi.org/10.4422/ ager.2013.01

Shah, T. I., Milosavljevic, S., y Bath, B. (2017). Measuring geographical accessibility to rural and remote health care services: Challenges and considerations. Spatial and Spatio-Temporal Epidemiology, 21, 87-96. https://doi.org/10.1016/j.sste.2017. 04.002

Shergold, I., Parkhurst, G., y Musselwhite, C. (2014). Rural car dependence: an emerging barrier to community activity for older people. Transportation Planning and Technology, 35(1), 69-85. https://doi.org/10.1080/03081 060.2012 .635417
Silva, C. (2013). Structural accessibility for mobility management. Progress in Planning, 81, 1-49. https://doi.org/10.1016/j.progress.2012.07.001

Soria-Lara, J.A., Banister, D. (2017). Dynamic participation processes for policy packaging in transport backcasting studies. Transport Policy, 58, 19-30. https://doi.org/10.1016/j.tranpol.2017.04.006

van Leeuwen, E. S., y Rietveld, P. (2011). Spatial Consumer Behaviour in Small and Medium-sized Towns. Regional Studies, 45(8), 1107-1119. https://doi.org/10.1080/00343401003713407

van Wee, B. (2016). Accessible accessibility research challenges. Journal of Transport Geography, 51, 9-16. https://doi.org/10.1016/j.jtrangeo.2015.10.018

Vega, A. (2012). Using Place Rank to measure sustainable accessibility. Journal of Transport Geography, 24, 411-418. https://doi.org/10.1016/j. jtrangeo.2012.04.008

Willis, K. S. (2019). Making a "Place" for ICTs in Rural Communities. Proceedings of the 9th International Conference on Communities y Technologies - Transforming Communities - C\&T '19, 136-142. https://doi.org/10.1145/3328320.3328401

Yeager, C. D., y Gatrell, J. D. (2014). Rural food accessibility: An analysis of travel impedance and the risk of potential grocery closures. Applied Geography, 53, 1-10. https://doi.org/10.1016/j. apgeog.2014.05.018

Zhen, F., Du, X., Cao, J., y Mokhtarian, P. L. (2018). The association between spatial attributes and e-shopping in the shopping process for search goods and experience goods: Evidence from Nanjing. Journal of Transport Geography, 66, 291-299. https://doi. org/10.1016/j.jtrangeo.2017.11.007 


\section{ANEXO 1}

ENCUESTA REALIZADA DURANTE LAS CAMPAÑAS DE CAMPO

\section{ACCESIBILIDAD A LA ACTIVIDAD COMERCIAL EN ZONAS DESPOBLADAS APLICACIÓN A LA COMARCA DEL MAESTRAZGO}

\section{BLOQUE 1: CARACTERÍSTICAS SOCIOECONÓMICAS}

Sexo: Mujer Hombre Edad: Municipio de residencia:

Nivel de Estudios: Sin Estudios Básico Secundaria Bachiller Universidad

¿Tiene una actividad laboral remunerada? Sí No

¿Dispone de carné de conducir? Sí No

¿Tiene coche? Sí No

¿Cuál es su renta mensual? Menos de $1.000 € \quad 1.000-2.000 € \quad 2.000-3.000 € \quad 3.000-5.000 €$ Más de $5.000 €$

\section{BLOQUE 2: ACCESIBILIDAD EN COCHE}

1. ¿Cuánto tiempo (en minutos) estaría dispuesto a conducir hasta el comercio diario? (Panadería, pescadería, frutería, supermercado)

Nada $15 \mathrm{~min} 30 \mathrm{~min} 45 \mathrm{~min}$ 1h. 1h. y media 2 horas 2 horas y media 3 horas o más

2. ¿Cuánto tiempo (en minutos) estaría dispuesto a conducir hasta el comercio semanal? (Peluquería, gimnasio, ferretería, perfumería)

Nada $15 \mathrm{~min} 30 \mathrm{~min} 45 \mathrm{~min}$ 1h. 1 h. y media 2 horas 2 horas y media 3 horas o más

3. ¿Cuánto tiempo (en minutos) estaría dispuesto a conducir hasta el comercio ocasional? (Tecnología, joyería, ropa, óptica)

Nada $15 \mathrm{~min} 30 \mathrm{~min} 45 \mathrm{~min}$ 1h. 1h. y media 2 horas 2 horas y media 3 horas o más

\section{BLOQUE 3: ACCESIBILIDAD EN TRANSPORTE PÚBLICO}

4. ¿Cuánto tiempo (en minutos) estaría dispuesto a ir en autobús hasta el comercio diario? (Panadería, pescadería, frutería, supermercado)

Nada $15 \mathrm{~min} 30 \mathrm{~min} 45 \mathrm{~min}$ 1h. 1 h. y media 2 horas 2 horas y media 3 horas o más

5. ¿Cuánto tiempo (en minutos) estaría dispuesto a ir en autobús hasta el comercio semanal? (Peluquería, gimnasio, ferretería, perfumería)

Nada $15 \mathrm{~min} 30 \mathrm{~min} 45 \mathrm{~min}$ 1h. 1h. y media 2 horas 2 horas y media 3 horas o más

6. ¿Cuánto tiempo (en minutos) estaría dispuesto a ir en autobús hasta el comercio ocasional? (Tecnología, joyería, ropa, óptica)

Nada $15 \mathrm{~min} 30 \mathrm{~min} 45 \mathrm{~min}$ 1h. 1 h. y media 2 horas 2 horas y media 3 horas o más

\section{BLOQUE 4: ACCESIBILIDAD NO MOTORIZADA}

7. ¿Cuánto tiempo (en minutos) estaría dispuesto a ir caminando hasta el comercio diario? (Panadería, pescadería, frutería, supermercado)

Nada $15 \mathrm{~min} 30 \mathrm{~min} 45 \mathrm{~min}$ 1h. 1h. y media 2 horas 2 horas y media 3 horas o más

8. ¿Cuánto tiempo (en minutos) estaría dispuesto a ir en bicicleta hasta el comercio diario? (Panadería, pescadería, frutería, supermercado)

Nada $15 \mathrm{~min} 30 \mathrm{~min} 45 \mathrm{~min}$ 1h. 1h. y media 2 horas 2 horas y media 3 horas o más 
9. ¿Cuánto tiempo (en minutos) estaría dispuesto a ir caminando hasta el comercio semanal? (Peluquería, gimnasio, ferretería, perfumería)

Nada $15 \mathrm{~min} 30 \mathrm{~min} 45 \mathrm{~min}$ 1h. 1h. y media 2 horas 2 horas y media 3 horas o más

10. ¿Cuánto tiempo (en minutos) estaría dispuesto a ir en bicicleta hasta el comercio semanal? (Peluquería, gimnasio, ferretería, perfumería)

Nada $15 \mathrm{~min} 30 \mathrm{~min} 45 \mathrm{~min}$ 1h. 1h. y media 2 horas 2 horas y media 3 horas o más

11. ¿Cuánto tiempo (en minutos) estaría dispuesto a ir en caminando hasta el comercio ocasional? (Tecnología, joyería, ropa, óptica)

Nada $15 \mathrm{~min} 30 \mathrm{~min} 45 \mathrm{~min}$ 1h. 1h. y media 2 horas 2 horas y media 3 horas o más

12. ¿Cuánto tiempo (en minutos) estaría dispuesto a ir en bicicleta hasta el comercio ocasional? (Tecnología, joyería, ropa, óptica)

Nada $15 \mathrm{~min} 30 \mathrm{~min} 45 \mathrm{~min}$ 1h. 1 h. y media 2 horas 2 horas y media 3 horas o más

BLOQUE 5: PREFERENCIA DE MODOS DE TRANSPORTE

13. Marque con una cruz el modo de transporte que prefiere para desplazarse a cada tipo de comercio

\begin{tabular}{|c|l|l|l|l|}
\hline & Caminando & Bicicleta & Coche & Transporte Público \\
\hline Diario & & & & \\
\hline No diario & & & & \\
\hline
\end{tabular}

\section{BLOQUE 6: ACCESIBILIDAD PERCIBIDA}

\section{Accesibilidad percibida en coche}

Rodee un número del 1 al 5 siendo 1 muy mala y 5 muy buena

¿Cómo percibe la accesibilidad en coche al comercio diario?

$\begin{array}{lllllll}\text { Muy mala } & 1 & 2 & 3 & 4 & 5 & \text { Muy buena }\end{array}$

¿Cómo percibe la accesibilidad en coche al comercio semanal?

$\begin{array}{lllllll}\text { Muy mala } & 1 & 2 & 3 & 4 & 5 & \text { Muy buena }\end{array}$

¿Cómo percibe la accesibilidad en coche al comercio ocasional?

$\begin{array}{lllllll}\text { Muy mala } & 1 & 2 & 3 & 4 & 5 & \text { Muy buena }\end{array}$

15. Accesibilidad percibida en transporte público

Rodee un número del 1 al 5 siendo 1 muy mala y 5 muy buena

¿Cómo percibe la accesibilidad en autobús al comercio diario?

$\begin{array}{lllllll}\text { Muy mala } & 1 & 2 & 3 & 4 & 5 & \text { Muy buena }\end{array}$

¿Cómo percibe la accesibilidad en autobús al comercio semanal?

$\begin{array}{lllllll}\text { Muy mala } & 1 & 2 & 3 & 4 & 5 & \text { Muy buena }\end{array}$

¿Cómo percibe la accesibilidad en autobús al comercio ocasional?

$\begin{array}{lllllll}\text { Muy mala } & 1 & 2 & 3 & 4 & 5 & \text { Muy buena }\end{array}$

16. Accesibilidad percibida caminando

Rodee un número del 1 al 5 siendo 1 muy mala y 5 muy buena 
¿Cómo percibe la accesibilidad caminando al comercio diario?

$\begin{array}{lllllll}\text { Muy mala } & 1 & 2 & 3 & 4 & 5 & \text { Muy buena }\end{array}$

¿Cómo percibe la accesibilidad caminando al comercio semanal? $\begin{array}{lllllll}\text { Muy mala } & 1 & 2 & 3 & 4 & 5 & \text { Muy buena }\end{array}$

¿Cómo percibe la accesibilidad caminando al comercio ocasional? $\begin{array}{lllllll}\text { Muy mala } & 1 & 2 & 3 & 4 & 5 & \text { Muy buena }\end{array}$

\section{Accesibilidad percibida en bicicleta}

Rodee un número del 1 al 5 siendo 1 muy mala y 5 muy buena

¿Cómo percibe la accesibilidad en bicicleta al comercio diario?

$\begin{array}{lllllll}\text { Muy mala } & 1 & 2 & 3 & 4 & 5 & \text { Muy buena }\end{array}$

¿Cómo percibe la accesibilidad en bicicleta al comercio semanal?

$\begin{array}{lllllll}\text { Muy mala } & 1 & 2 & 3 & 4 & 5 & \text { Muy buena }\end{array}$

¿Cómo percibe la accesibilidad en bicicleta al comercio ocasional?

$\begin{array}{lllllll}\text { Muy mala } & 1 & 2 & 3 & 4 & 5 & \text { Muy buena }\end{array}$ 